\title{
A New Explanation of Inflammation in Rheumatoid Arthritis Patients With Respect to Claudin-5, Matrix Metalloproteinase-9, and Neuroserpin
}

\author{
Sevil Arabacı TAMER, ${ }^{1}$ Gönül GÜROL, ${ }^{2}$ İbrahim TEKEOĞLU, ${ }^{3}$ \\ Halil HARMAN, ${ }^{3}$ İhsan Hakkı ÇífTÇİ ${ }^{4}$ \\ ${ }^{1}$ Department of Physiology, Medical Faculty of Marmara University, Istanbul, Turkey \\ ${ }^{2}$ Department of Physiology, Medical Faculty of Sakarya University, Sakarya, Turkey \\ ${ }^{3}$ Department of Physical Medicine and Rehabilitation, Division of Rheumatology, Medical Faculty of Sakarya University, Sakarya, Turkey \\ ${ }^{4}$ Department of Microbiology, Medical Faculty of Sakarya University, Sakarya, Turkey
}

\begin{abstract}
Objectives: This study aims to investigate the relationship between neuroserpin (NSP) and claudin-5, as well as matrix metalloproteinase-9 (MMP-9), with respect to clinical activity of disease in patients with rheumatoid arthritis.

Patients and methods: The study included a total of 75 patients (18 males, 57 females; mean age $48.12 \pm 11.23$ years; range 20 to 60 years) who were admitted to the rheumatology outpatient facility at the Medical Faculty Hospital, Sakarya University, in October 2014. Patients were divided into four groups based on their Disease Activity Score 28 (DAS28) scores as remission group ( $n=16$, DAS28 $<2.6$ ), low disease activity group ( $n=16$, DAS28 between 2.6-3.2), moderate disease activity group ( $n=28$, DAS28 between 3.2-5.1), and high disease activity group $(n=15$, DAS28 $>5.1)$. Ten healthy subjects (HS) served as controls.

Results: Claudin-5, MMP-9, and NSP levels were significantly different in rheumatoid arthritis patients compared to HS ( $p=0.035,0.026$, and 0.014 , respectively). Additionally, there were no differences between claudin-5 levels and disease activity among all RA groups. However, compared to HS, patient groups showed a significant difference $(p=0.035)$ in terms of claudin- 5 levels. Serum levels of MMP-9 were significantly different in moderate disease activity group compared to HS $(p=0.013)$. Levels of NSP were significantly different in moderate disease activity and high disease activity groups compared to HS ( $\mathrm{p}=0.008$ and 0.031 , respectively).

Conclusion: Our study demonstrated the differential associations of endothelial function/dysfunction biomarkers and disease activity in rheumatoid arthritis. How and why this impairment occurs is not fully understood and more data regarding NSP, MMP, and claudin expression in plasma are warranted.

Keywords: Claudin-5; matrix metalloproteinase-9; neuroserpin; rheumatoid arthritis.
\end{abstract}

Rheumatoid arthritis (RA) is a major autoimmune disease affecting $1 \%$ to $2 \%$ of the adult population. $\mathrm{RA}$ is characterized by synovial joint inflammation with accompanying destruction of joint. ${ }^{1,2}$ Because $\mathrm{RA}$ is a chronic inflammatory disease of the joints, plasma levels of inflammatory cytokines, such as interleukin (IL)-6, IL-17, IL-22, and IL-1 $\beta$, and tumor necrosis factor-alpha, are increased in both RA patients and animal models. ${ }^{3,4}$ In addition, synovial inflammation in RA causes increased cytokine release and abnormally high levels of acute reactive proteins, such as $\mathrm{C}$-reactive protein (CRP). ${ }^{5}$

Endothelial dysfunction (ED) is characterized by a shift of actions by the endothelium towards reduced vasodilation, a proinflammatory and proadhesive state, and prothrombic properties. Therefore, several biological markers, which serve

Received: February 17, 2016 Accepted: April 03, 2016 Published online: August 01, 2016

Correspondence: Gönül Gürol, MD. Sakarya Üniversitesi Tıp Fakültesi Fizyoloji Anabilim Dalı, 54050 Korucuk, Sakarya, Turkey.

Tel: +90 264 - 2955454 e-mail: gonulgurol@yahoo.com

○2016 Turkish League Against Rheumatism. All rights reserved. 
as ED indicators, are used to assess the targets of each vascular phenotype. ${ }^{6}$ The presence of ED is a characteristic feature of patients with RA. ${ }^{7}$ ED markers include endothelin-1, von Willebrand factor, tissue plasminogen activator (tPA), plasminogen activator inhibitor, and adhesion molecules. ${ }^{6}$ Poredos ${ }^{8}$ showed that the cause of ED in RA may be systemic high-grade inflammation. In addition, asymmetric dimethylarginine, which is an endogenous competitive inhibitor of nitric oxide synthase, has been associated with ED. ${ }^{9}$ Tight junctions (TJs) also play a critical role in epithelial barrier function that involve complexes between adjacent epithelial cells, control paracellular passage, and maintain cell polarity. ${ }^{10,11}$ TJs exist in several cerebral vascular endothelial cells, such as zonula occludens-1, occluding, and the claudin family. ${ }^{12}$ However, endothelial barrier function requires the adhesive activity of both E-cadherin and claudin-5 (Cld-5). ${ }^{13}$ Collagen-induced arthritis may cause bloodbrain barrier (BBB) hyperpermeability and alter the expression of $\mathrm{TJ}$ proteins, including Cld-5. Moreover, the integrity of the $\mathrm{BBB}$ is damaged by ED. ${ }^{14}$

Neuroserpin (NSP) is a serine protease inhibitor and member of the serpin family. It is expressed in developing and adult nervous systems, and acts as an inhibitor of protease tPA and a regulator of neuronal growth and plasticity. ${ }^{15,16}$ By means of complex formation with tPA, NSP restricts microglial activation; hence, the production of inflammatory mediators decreases, as reflected by reduced serum levels. ${ }^{17}$ In other words, NSP displays anti-inflammatory activity. ${ }^{18}$ tPA can also activate matrix metalloproteinases (MMPs), specifically MMP-9. MMPs are a family of endopeptidases that primarily catalyze the degradation of the extracellular matrix (ECM), and in turn, increase BBB permeability. During various physiological and pathological events, such as embryogenesis, ovulation, arthritis, and inflammatory conditions, MMP production is stimulated. ${ }^{19-21}$ In ischemia-induced brain injury, abnormal upregulation of MMP-9 has been linked to $\mathrm{BBB}$ disruption by ECM degradation, basal lamina proteins, and TJs surrounding the BBB. Ischemic injury induces significantly decreased expression of Cld-5, which disrupts BBB integrity and increases MMP-9 expression. ${ }^{22}$ MMPs also play critical roles in RA, and the expression of specific members of the MMP family, including MMP-3, MMP-8, and MMP-9, is significantly related to disease advancement. ${ }^{23}$ In this regard, MMP-9 is detected at high levels in active RA patients. ${ }^{24}$ However, to the best of our knowledge, there is no combined study that focuses on MMP-9 and Cld- 5 in RA patients. Therefore, in this study, we aimed to investigate the relationship between NSP and Cld-5, as well as MMP-9, with respect to clinical activity of disease in patients with RA.

\section{PATIENTS AND METHODS}

The study included a total of 75 patients (18 males, 57 females; mean age 48.12 \pm 11.23 years; range 20 to 60 years) who were admitted to the rheumatology outpatient facility at the Medical Faculty Hospital, Sakarya University, in October 2014. RA diagnosis was based on the diagnostic criteria revised in 2010 by the American College of Rheumatology/European League Against Rheumatism. ${ }^{25}$ Ten healthy subjects (HS) admitted to our outpatient hospital for a checkup served as controls. Patients who (i) did not meet the criteria for RA; (ii) were treated for another rheumatic disease in the past; (iii) had a history of diabetes mellitus, renal failure, or liver failure; and (iv) were on tumor necrosis factor blockers were excluded. Disease activity was determined by Disease Activity Score 28 (DAS28), which was calculated using results from clinical and laboratory data. DAS28 remission criteria, including levels of CRP, swollen and tender joint counts, and global health assessment, were used to determine whether the disease was in remission. A DAS28 score between 2.6 to 3.2 indicates low disease activity, 3.2 to 5.1 indicates moderate activity, and $>5.1$ indicates high disease activity. ${ }^{26}$

Patients were then divided into four groups based on their DAS28 scores as remission group (RG) ( $n=16$, DAS28 <2.6), low disease activity group (LDAG) ( $\mathrm{n}=16$, DAS28 between 2.6-3.2), moderate disease activity group (MDAG) $(n=28$, DAS28 between 3.2-5.1), and high disease activity group (HDAG) ( $\mathrm{n}=15$, DAS28 $>5.1$ ). Patients' demographic data, including age, sex, smoking habit, and clinical data such as duration of symptoms, duration of disease, and delay in diagnosis were recorded. 
Rheumatoid factor (RF) was measured by nephelometry (Beckman Coulter IMMAGE ${ }^{\circledR}$ 800 , USA); levels greater than $20 \mathrm{U} / \mathrm{mL}$ were considered positive. Anti-cyclic citrullinated peptide (anti-CCP) levels were measured using an enzyme linked immunosorbent assay (Abbott Laboratories. Abbott Park, IL, USA); those greater than $0.5 \mathrm{U} / \mathrm{mL}$ were considered positive. NSP (Hangzhou Eastbiopharm Co., Ltd, China), MMP-9 (eBioscience, San Diego, California, USA) and Cld-5 (Eastbiopharm, China) were assessed by enzyme linked immunosorbent assay from serum samples.

Ethics approval was obtained from the local Ethics Committee of Sakarya University (approval number: 71522473/050.01.04/86). A written informed consent was obtained from each patient. The study was conducted in accordance with the principles of the Declaration of Helsinki.

\section{Statistical analysis}

Statistical analysis was performed using IBM SPSS software, version 20.0 (IBM Corporation, Armonk, NY, USA). Values are presented as the mean \pm standard deviation and interquartile range. Differences between groups and HS were determined by one-way analysis of variance. $P$ values less than 0.05 were considered statistically significant.

\section{RESULTS}

Age, sex, and smoking habits were similar between RA patients and HS. Average disease duration, duration of symptoms, and delay in diagnosis were $43.56 \pm 36.95$ months, $51.01 \pm 38.18$ months, and $7.77 \pm 5.01$ months, respectively. These results and clinical characteristics of RA patients are summarized in Table 1 . With respect to diseasemodifying anti-rheumatic drugs, disease duration and duration of symptoms were determined to be significantly different among RA groups $(p<0.001)$; however, delay in diagnosis did not significantly differ among RA groups (Table 2).

A significant difference was observed between HS and RA patients in terms of erythrocyte sedimentation rate values $(p<0.001)$. Within RA groups, CRP values were significantly different. Moreover, a significant difference was revealed among MDAG individuals and HS $(p=0.001)$. Patients with RA had significantly higher RF values $(p<0.001)$ than HS. Anti-CCP levels of remission group compared to MDAG were different, but not statistically significant $(p=0.071)$. In addition, antiCCP levels were significantly different $(p=0.008)$ in HS compared to patient groups.

Our results revealed no differences between Cld-5 levels and disease activity. However, when patient groups were compared to HS, a significant difference was detected $(p=0.035)$. Figure 1a shows the relationship between Cld- 5 and stage of disease activity. Serum levels of MMP-9 were significantly different in MDAG patients compared to HS $(p=0.013)$. MMP-9 in HDAG patients was only moderately different from HS ( $p=0.055)$, whereas all RA groups were significantly different from HS $(p=0.026)$. Figure $1 b$ shows the relationship between MMP-9 and stage of disease activity. When HS were compared to MDAG and HDAG patients, serum levels of NSP were significantly different ( $p=0.008$ and 0.031 , respectively). All RA groups were significantly different from HS in terms of serum levels of NSP $(p=0.014)$. Figure 1c shows the relationship between NSP levels and stage of disease activity.

Table 1. Demographic and clinical characteristics of study groups

\begin{tabular}{|c|c|c|c|c|}
\hline \multirow[t]{2}{*}{ Characteristics } & \multicolumn{2}{|c|}{ Rheumatoid arthritis patients } & \multicolumn{2}{|c|}{ Healthy subjects } \\
\hline & $\%$ & Mean $\pm \mathrm{SD}$ & $\%$ & Mean \pm SD \\
\hline Age (years) & & $48.1 \pm 11.2$ & & $46.2 \pm 12.4$ \\
\hline Gender (female) & 76.0 & & 60.0 & \\
\hline Cigarette smoking & 34.7 & & 40.0 & \\
\hline DS (months) & & $51.0 \pm 38.2$ & & NA \\
\hline DD (months) & & $43.6 \pm 37.0$ & & NA \\
\hline DED (months) & & $7.8 \pm 5.0$ & & NA \\
\hline Health Assessment Questionnaire total scores & & $2.7 \pm 0.1$ & & NA \\
\hline
\end{tabular}




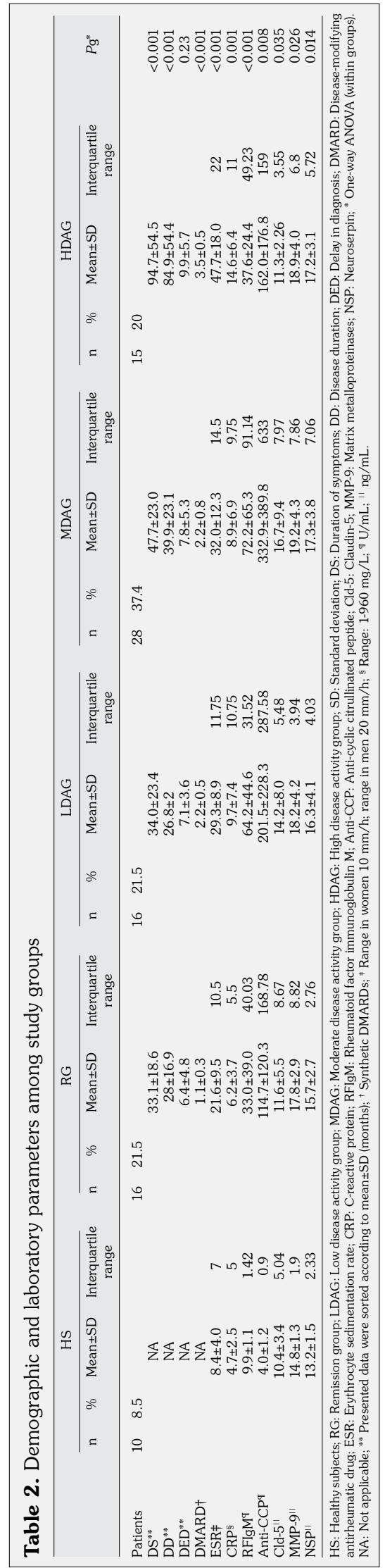

\section{DISCUSSION}

To the best of our knowledge, this is the first study demonstrating that Cld-5, MMP-9, and NSP levels are significantly different in RA patients relative to HS ( $p=0.035,0.026$, and 0.014 , respectively). NSP is a member of the serine protease inhibitor or serpin superfamily of proteins. ${ }^{15}$ Thrombolytic proteases, their receptors, and serine protease inhibitors (serpins) play an important role as regulatory counterbalances in homeostasis and vascular inflammation. It has been suggested that NSP inhibits tPA and urokinase plasminogen activator (uPA), in order of descending inhibitory activity. ${ }^{18}$ In a previous study, plasma soluble uPA receptor concentrations were evaluated in RA patients. Soluble uPA receptor is believed to be particularly valuable in recognition of inflammatory activity in patients in remission, according to DAS28 scores. ${ }^{27,28}$ However, increased levels of UPA and PA inhibitors, such as plasminogen activator inhibitor -1 and -2 , are also found in synovial tissue in RA patients. ${ }^{29}$ In contrast, Cook et al. ${ }^{30}$ indicated that the major effect of uPA in the collagen-induced arthritis model is deleterious, whereas that of tPA is protective. These data and the complexities of PA function highlight that approaches targeting uPA or aimed towards enhancing local tPA activity in joints may be of therapeutic benefit in RA. In our study, NSP levels, as well as CRP and erythrocyte sedimentation values, were elevated in patients with RA compared to HS.

Our findings also suggest that NSP may serve as an inflammatory marker in RA patients. In the literature, there is some evidence of a relationship between serine proteases in clot-forming (thrombotic) and clot-dissolving (thrombolytic) cascades, activated by an inflammatory cytokine storm, which can induce systemic inflammation with a loss of normal serine protease inhibitor (serpin) function. ${ }^{31}$ In addition, NSP possesses anti-inflammatory activity in systemic arteries, modifies $\mathrm{T}$ helper cell responses, and significantly reduces plaque formation in mouse aortic allografts. ${ }^{18}$

We found an association between anti$\mathrm{CCP} / \mathrm{RF}$ immunoglobulin $\mathrm{M}$ levels and $\mathrm{Cld}-5$ in MDAG patients based on DAS28 scores. This is supported by several earlier studies suggesting that the presence of anti-CCP antibodies and 
(a)

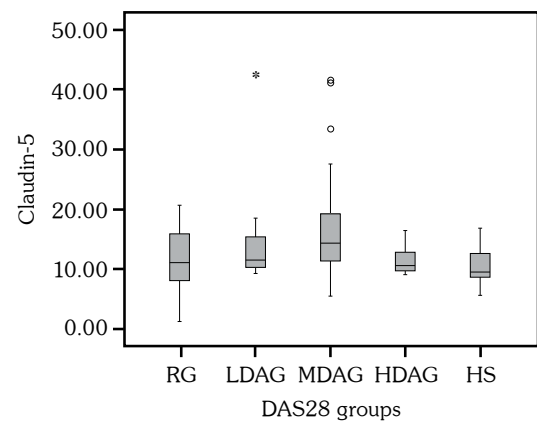

(b)

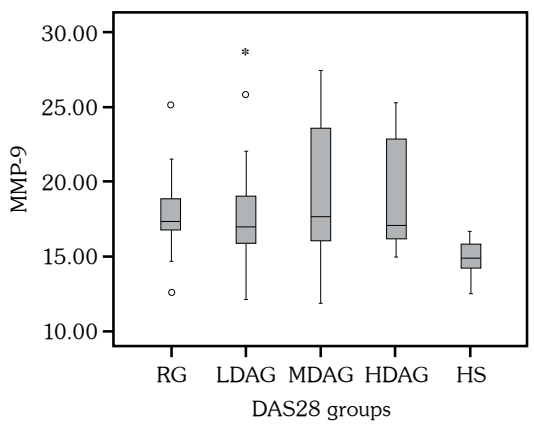

(c)

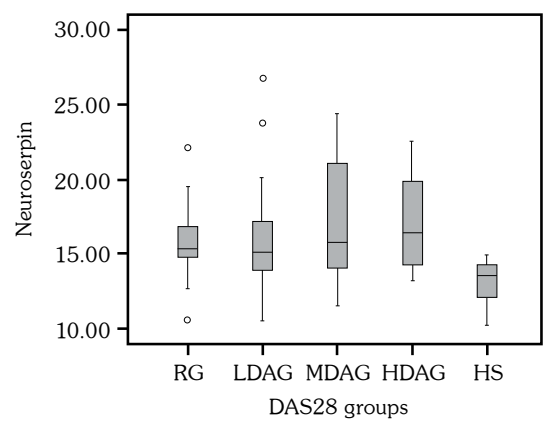

Figure 1. Relationship between (a) claudin-5, (b) MMP-9 and (c) neuroserpin levels and disease activity. MMP: Matrix metalloproteinases; RG: Remission group; LDAG: Low disease activity group; MDAG: Moderate disease activity group; HDAG: High disease activity group; HS: Healthy subjects; DAS28: Disease Activity Score 28.

$\mathrm{RF}$ immunoglobulin $\mathrm{M}$ is related to impaired endothelial function independent of cardiovascular risk factors in patients with RA. ${ }^{32}$

Physiologic and pathophysiologic events in tissue alter endothelial cell interactions with soluble and cellular blood components. Recent studies with RA patients have indeed revealed interactions between $\mathrm{ED}$ and disease activity; these include soluble human vascular cell adhesion protein 1 , von Willebrand factor, pentraxin-3, asymmetric dimethyl-L-arginine, soluble E-selectin, monocyte chemotactic protein-1, and osteoprotegerin. ${ }^{33}$ Many of these substances are affected by inflammatory mediators and cytokines, and as specified earlier, contribute to the mechanisms underlying $\mathrm{RA}$ pathogenesis. With respect to therapeutic strategies for RA treatment, especially anti-tumor necrosis factor-alpha therapies, most are effective at reducing inflammation, as well as improving endothelial function. ${ }^{34}$ In this regard, we found higher Cld-5 and NSP levels in MDAG patients compared to HDAG individuals.

After exposure to inflammatory stimuli, endothelial cells in virtually every tissue express plasminogen activator inhibitor- $1 .{ }^{35}$ In our previous study, we demonstrated increased von Willebrand factor and Cld-5. ${ }^{36}$ It is also important to note higher levels of Cld-5, MMP-9, and anti-CCP in MDAG individuals relative to other RA subgroups. There are several potential explanations for these findings. Firstly, this could be due to circulating Cld-5 and MMP, or autoimmunity that has not yet developed in individuals of remission and low disease activity groups. Alternatively, this result may indicate that Cld-5 and/or MMP-9 are in the circulation in the absence of clinically apparent RA in patients who have demonstrated high disease activity based on their DAS28 scores. Furthermore, our study demonstrated the complex regulation of Cld-5 and intricate balance of protein expression for disease activity in RA patients. Studies with various inducers of inflammatory pain have highlighted similar regulation of $\mathrm{TJ}$ proteins. Studies are also needed to evaluate the potential mechanisms of RA-related disease activity responses, including NSP and Cld-5, in brain endothelial cells. Informative animal models will also be important to understand how RA-related disease activity may alter Cld-5 and NSP levels.

Recent studies have investigated the relationship between MMP family members, plasminogen activators and their inhibitors, and RA. MMPs, which are produced primarily in fibroblast-like synoviocytes, are proteases that participate in the irreparable proteolytic degradation and remodeling of the ECM. Activation of inactive pro-MMPs is dependent on other regulatory mechanisms, including the plasminogen-plasmin cascade, which is itself regulated by serine proteinases uPA and tPA. A recent study demonstrated that the level of MMP-9, which is strongly associated with uPA, is increased in the synovial fluid of RA patients. ${ }^{37}$ In RA, an imbalance between inhibition and activation of MMPs secreted by endothelial cells can result in excessive degradation of the ECM. ${ }^{38}$ Consistent with this report, the level of NSP, a natural inhibitor of tPA, was significantly increased in RA and strongly associated with the level of MMP-9 in RA patients in our study. 
We may speculate that NSP may be involved, at least in part, in the pathogenesis of arthritis through activation of MMP-9. This hypothesis is in agreement with previous studies that have described the involvement of serine proteinases and cysteine proteinases in MMP activation and collagen degradation, respectively. ${ }^{39}$ However, because tPA can also activate MMPs, we should also consider that an increase in NSP observed between RA patients and HS may involve MMP-9, albeit directly or indirectly (via inflammationassociated mechanisms). We acknowledge that the major limitation of our study was that proinflammatory mediators and cytokines, which are abundantly produced in RA and exert numerous effects in endothelial cells, were not assayed.

In conclusion, our study demonstrates differential associations of endothelial function/ dysfunction biomarkers and disease activity in RA. Cld-5, MMP-9 and NSP levels are increased in patients with RA compared to healthy controls. However, higher levels of Cld-5, MMP-9, and NSP were also observed in patients with MDAG. Nevertheless, how and why this impairment occurs is not fully understood, and more data regarding NSP, MMP, and claudin expression in plasma are warranted.

\section{Declaration of conflicting interests}

The authors declared no conflicts of interest with respect to the authorship and/or publication of this article.

\section{Funding}

The authors thanks the Sakarya University Research Project Committee for financial support of the project (Project Number: 2014-80-01-002).

\section{REFERENCES}

1. Ahmad SF, Zoheir KM, Bakheet SA, Ashour AE, Attia SM. Poly(ADP-ribose) polymerase-1 inhibitor modulates $\mathrm{T}$ regulatory and IL-17 cells in the prevention of adjuvant induced arthritis in mice model. Cytokine 2014;68:76-85.

2. Brahn E, Lee S, Lucas A, McFadden G, Macaulay C. Suppression of collagen-induced arthritis with a serine proteinase inhibitor (serpin) derived from myxoma virus. Clin Immunol 2014;153:254-63.

3. Okazaki H, Lin Q, Nishikawa K, Ohtsuji N, Tsurui H, Ohtsuji M, et al. TNF $\alpha$ but not IL-17 is critical in the pathogenesis of rheumatoid arthritis spontaneously occurring in a unique Fc $\gamma$ RIIB-deficient mouse model.
Mod Rheumatol 2014;24:931-8.

4. Choi JH, Lee JH, Roh KH, Seo SK, Choi IW, Park $\mathrm{SG}$, et al. Gallium nitrate ameliorates type II collageninduced arthritis in mice. Int Immunopharmacol 2014;20:269-75.

5. Riegsecker S, Wiczynski D, Kaplan MJ, Ahmed S. Potential benefits of green tea polyphenol EGCG in the prevention and treatment of vascular inflammation in rheumatoid arthritis. Life Sci 2013;93:307-12.

6. Poredos P. Endothelial dysfunction in the pathogenesis of atherosclerosis. Int Angiol 2002;21:109-16.

7. Totoson P, Maguin-Gaté K, Prati C, Wendling D, Demougeot $C$. Mechanisms of endothelial dysfunction in rheumatoid arthritis: lessons from animal studies. Arthritis Res Ther 2014;16:202.

8. Poredos P. Endothelial dysfunction in the pathogenesis of atherosclerosis. Clin Appl Thromb Hemost 2001;7: 276-80.

9. Chen $\mathrm{YH}, \mathrm{Xu} \mathrm{X}$, Sheng $\mathrm{MJ}$, Zheng Z, Gu Q. Effects of asymmetric dimethylarginine on bovine retinal capillary endothelial cell proliferation, reactive oxygen species production, permeability, intercellular adhesion molecule-1, and occludin expression. Mol Vis 2011;17:332-40.

10. Hou J. The kidney tight junction (Review). Int $\mathrm{J}$ Mol Med 2014;34:1451-7.

11. Lu RY, Yang WX, Hu YJ. The role of epithelial tight junctions involved in pathogen infections. Mol Biol Rep 2014;41:6591-610.

12. Ma X, Zhang H, Pan Q, Zhao Y, Chen J, Zhao B, et al. Hypoxia/Aglycemia-induced endothelial barrier dysfunction and tight junction protein downregulation can be ameliorated by citicoline. PLoS One 2013;8:e82604.

13. Gavard J, Gutkind JS. VE-cadherin and claudin-5: it takes two to tango. Nat Cell Biol 2008;10:883-5.

14. Nishioku T, Yamauchi A, Takata F, Watanabe T, Furusho K, Shuto $\mathrm{H}$, et al. Disruption of the bloodbrain barrier in collagen-induced arthritic mice. Neurosci Lett 2010;482:208-11.

15. Galliciotti G, Sonderegger P. Neuroserpin. Front Biosci 2006;11:33-45.

16. Sarkar A, Zhou C, Meklemburg R, Wintrode PL. Local conformational flexibility provides a basis for facile polymer formation in human neuroserpin. Biophys J 20115;101:1758-65.

17. Rodríguez-González R, Sobrino T, Rodríguez-Yáñez M, Millán M, Brea D, Miranda E, et al. Association between neuroserpin and molecular markers of brain damage in patients with acute ischemic stroke. J Transl Med 2011;9:58.

18. Munuswamy-Ramanujam G, Dai E, Liu L, Shnabel M, Sun YM, Bartee M, et al. Neuroserpin, a thrombolytic serine protease inhibitor (serpin), blocks transplant vasculopathy with associated modification of T-helper cell subsets. Thromb Haemost 2010;103:545-55.

19. Adibhatla RM, Hatcher JF. Tissue plasminogen activator (tPA) and matrix metalloproteinases in the 
pathogenesis of stroke: therapeutic strategies. CNS Neurol Disord Drug Targets 2008;7:243-53.

20. Lakhan SE, Kirchgessner A, Tepper D, Leonard A. Matrix metalloproteinases and blood-brain barrier disruption in acute ischemic stroke. Front Neurol 2013;4:32

21. Sapna G, Gokul S, Bagri-Manjrekar K. Matrix metalloproteinases and periodontal diseases. Oral Dis 2014;20:538-50.

22. Bai X, Zhang X, Chen L, Zhang J, Zhang L, Zhao X, et al. Protective effect of naringenin in experimental ischemic stroke: down-regulated NOD2, RIP2, NF-кB, MMP-9 and up-regulated claudin-5 expression. Neurochem Res 2014;39:1405-15.

23. Zhou M, Qin S, Chu Y, Wang F, Chen L, Lu Y. Immunolocalization of MMP-2 and MMP-9 in human rheumatoid synovium. Int $\mathrm{J}$ Clin Exp Pathol 2014;7:3048-56.

24. Kotani T, Takeuchi T, Takai S, Yoshida S, Hata K, Nagai K, et al. Serum levels of matrix metalloproteinase (MMP) 9, a risk factor for acute coronary syndrome, are reduced independently of serum MMP-3 by anti-TNF- $\alpha$ antibody (infliximab) therapy in patients with rheumatoid arthritis. J Pharmacol Sci 2012;120:50-3.

25. Aletaha D, Neogi T, Silman AJ, Funovits J, Felson DT, Bingham CO 3rd, et al. 2010 Rheumatoid arthritis classification criteria: an American College of Rheumatology/European League Against Rheumatism collaborative initiative. Arthritis Rheum 2010;62:2569-81.

26. Wells G, Becker JC, Teng J, Dougados M, Schiff M, Smolen $\mathrm{J}$, et al. Validation of the 28-joint Disease Activity Score (DAS28) and European League Against Rheumatism response criteria based on C-reactive protein against disease progression in patients with rheumatoid arthritis, and comparison with the DAS28 based on erythrocyte sedimentation rate. Ann Rheum Dis 2009;68:954-60.

27. Slot O, Brünner N, Locht $\mathrm{H}$, Oxholm P, Stephens RW. Soluble urokinase plasminogen activator receptor in plasma of patients with inflammatory rheumatic disorders: increased concentrations in rheumatoid arthritis. Ann Rheum Dis 1999;58:488-92.

28. Toldi G, Bekö G, Kádár G, Mácsai E, Kovács L, Vásárhelyi $\mathrm{B}$, et al. Soluble urokinase plasminogen activator receptor (suPAR) in the assessment of inflammatory activity of rheumatoid arthritis patients in remission. Clin Chem Lab Med 2013;51:327-32.

29. Busso N, Péclat V, So A, Sappino AP. Plasminogen activation in synovial tissues: differences between normal, osteoarthritis, and rheumatoid arthritis joints. Ann Rheum Dis 1997;56:550-7.

30. Cook AD, Braine EL, Campbell IK, Hamilton JA. Differing roles for urokinase and tissue-type plasminogen activator in collagen-induced arthritis. Am J Pathol 2002;160:917-26.

31. Chen H, Zheng D, Abbott J, Liu L, Bartee MY, Long $\mathrm{M}$, et al. Myxomavirus-derived serpin prolongs survival and reduces inflammation and hemorrhage in an unrelated lethal mouse viral infection. Antimicrob Agents Chemother 2013;57:4114-27.

32. Hjeltnes G, Hollan I, Førre Ø, Wiik A, Mikkelsen $\mathrm{K}$, Agewall S. Anti-CCP and RF IgM: predictors of impaired endothelial function in rheumatoid arthritis patients. Scand J Rheumatol 2011;40:422-7.

33. Klimek E, Skalska A, Kwasny-Krochin B, Surdacki A, Sulicka J, Korkosz M, et al. Differential associations of inflammatory and endothelial biomarkers with disease activity in rheumatoid arthritis of short duration. Mediators Inflamm 2014;2014:681635.

34. Spinelli FR, Metere A, Barbati C, Pierdominici M, Iannuccelli C, Lucchino B, et al. Effect of therapeutic inhibition of TNF on circulating endothelial progenitor cells in patients with rheumatoid arthritis. Mediators Inflamm 2013;2013:537539.

35. Sawdey MS, Loskutoff DJ. Regulation of murine type 1 plasminogen activator inhibitor gene expression in vivo. Tissue specificity and induction by lipopolysaccharide, tumor necrosis factor-alpha, and transforming growth factor-beta. $\mathrm{J}$ Clin Invest 1991;88:1346-53.

36. Gurol G, Ciftci IH, Harman H, Karakece E, Kamanli A, Tekeoglu I. Roles of claudin-5 and von Willebrand factor in patients with rheumatoid arthritis. Int J Clin Exp Pathol 2015;8:1979-84.

37. Kim KS, Lee YA, Choi HM, Yoo MC, Yang HI. Implication of MMP-9 and urokinase plasminogen activator (uPA) in the activation of pro-matrix metalloproteinase (MMP)-13. Rheumatol Int 2012;32:3069-75.

38. Lee WS, Lim JH, Sung MS, Lee EG, Oh YJ, Yoo WH. Ethyl acetate fraction from Angelica sinensis inhibits IL-1 $\beta$-induced rheumatoid synovial fibroblast proliferation and COX-2, PGE2, and MMPs production. Biol Res 2014;47:41.

39. Milner JM, Cawston TE. Matrix metalloproteinase knockout studies and the potential use of matrix metalloproteinase inhibitors in the rheumatic diseases. Curr Drug Targets Inflamm Allergy 2005;4:363-75. 